\title{
Structure of multispecies charged particles in a quadratic trap
}

\author{
Y. H. Liu, ${ }^{1,2}$ Z. Y. Chen, ${ }^{1}$ M. Y. Yu, ${ }^{3}$ L. Wang, ${ }^{2}$ and A. Bogaerts ${ }^{1}$ \\ ${ }^{1}$ Department of Chemistry, University of Antwerp, Universiteitsplein 1, B-2610 Antwerp, Belgium \\ ${ }^{2}$ Institute of Physics, Chinese Academy of Sciences, 100080 Beijing, Peaple's Republic of China \\ ${ }^{3}$ Theoretische Physik I, Ruhr-Universität Bochum, D-44780 Bochum, Germany
}

(Received 31 October 2005; published 17 April 2006)

\begin{abstract}
Multispecies interacting charged particles in a two-dimensional quadratic trap are studied. The ground-state configurations for different particle and species numbers are obtained by molecular dynamics simulation. It is found that particles with similar mass-to-charge ratio tend to populate a common shell, whose location depends on the particle mass-to-charge ratio, and that the greater the latter, the closer are the particles to the center of the trap. This scaling for the ground-state configuration is independent of the total particle and species numbers in the system.
\end{abstract}

DOI: 10.1103/PhysRevE.73.047402

PACS number(s): 36.40.Wa, 52.27.Lw, 61.43.Bn, 61.50.Ah

Two-dimensional (2D) charged-particle clusters in confined traps have been of considerable interest, since they can model electrons in quantum dots [1] and on the surface of liquid helium [2], vortices in superfluids [3], colloidal particles in traps [4], confined ferromagnetic particles [5-7], as well as dust grains in plasmas [8-11]. When confined to a 2D trap, the particles can appear in a lattice structure with the inner particles forming a roughly triangular lattice and the outer ones forming concentric rings that become more and more circular, conforming to the symmetry of the trap [12-14]. Such atomlike configurations have been studied in considerable detail by Schweigert et al. [15,16], and a practical Mendeleev-like table for the structures has been constructed.

Recently, Drocco et al. [17] investigated the structure and melting of 2D clusters consisting of two kinds of charged particles of the same mass, but one species has a charge twice the other. The particles were found to arrange themselves into concentric rings, with those having the larger charge located at the outer edge of the trap. Nelissen et al. [18] studied the ground states of a 2D charged-particle system containing one or two particles of different mass and charge, and showed that the ground state depends strongly on the charge and mass of these particle(s). More recently, Ferreira et al. [19] investigated a true 2D binary system, and showed that in the ground state similar particles tend to group together, but mixing of particles in the shells can appear if the mass and charge ratios of the corresponding species are equal. On the other hand, Matthey et al. [20] investigated a three-dimensional (3D) system containing two species of particles with the identical mass-to-charge ratio by molecular dynamics (MD) simulation, and found that the two species can mix completely independent of the relative abundances. However, a difference of one per thousand in the mass-to-charge ratios is sufficient to lead to segregation of the two species. Hornekær et al. [21] experimentally studied a 3D two-component $\left({ }^{40} \mathrm{Ca}^{+}\right.$and $\left.{ }^{24} \mathrm{Mg}^{+}\right)$ionic crystal confined in a Paul trap, and found radial separation of the two ion species. In another context, Grzybowski et al. [6,7] studied experimentally a 2D system with two different-sized ferromagnetic particles (disks) on liquid-air interface and confined by an external magnetic field. They found that different-sized particles can either separate or mix, depending on the experimental condition. In all the above studies, only two species of particles are considered, and how different species separate or mix is still not known in general. In practice, however, systems can contain many different species of different properties. It is thus of interest to investigate the ground-state structure of systems containing more than two species of charged particles and obtain the general conditions for species separation.

In this paper, we extend the work of Nelissen et al. [17-19] and study by MD simulation the structures of systems with several species of charged particles in a $2 \mathrm{D}$ quadratic trap, such as that of charged dust grains in a rotating plasma. The ground-state (the state at zero temperature) configurations of systems with different species and particle numbers are investigated and compared. It is found that particles with the same mass-to-charge ratio $s$ tend to group together in a common shell whose location also strongly depends on $s$ : the greater the value of $s$, the closer is the corresponding shell to the center of the trap. The rule also appears to be independent of the total particle number and species numbers.

We consider a system of $N=\sum_{k=1}^{n} N^{(k)}$ charged particles interacting through the $1 / r$ Coulomb potential, where $n$ is the total species number in the system, and $N^{(k)}$ is the particle number of the species $k$. We assume that the particles are confined by a quadratic trap $[8,10,12,13,17,18,22]$, centered at the origin and increasing radially as $r^{2}$. The Hamiltonian of the system is then $H=K+U$, where $K$ is the kinetic energy and

$$
U=\frac{1}{\varepsilon_{i<j}} \sum_{i<j}^{N} \frac{Q_{i}^{(k)} Q_{j}^{(k)}}{\left|\mathbf{r}_{i}-\mathbf{r}_{j}\right|}+\frac{1}{2} \sum_{i=1}^{N} M_{i}^{(k)} \omega_{0}^{2} r_{i}^{2},
$$

is the potential energy. In Eq. (1), $\mathbf{r}_{i}$ is the position of the particle $i(=1, \ldots, N), \omega_{0}$ is the constant trapping frequency, and $\varepsilon$ (constant) is the dielectric constant of the background medium. Moreover, $Q_{i}^{(k)}$ and $M_{i}^{(k)}$ are the effective charge and mass of the particle $i$ belonging to species $k$, respectively. In the ground state, we have $H \rightarrow U$. The potential energy can be rewritten as 


$$
U=\sum_{i<j}^{N} \frac{q_{i}^{(k)} q_{j}^{(k)}}{\left|\mathbf{r}_{i}-\mathbf{r}_{j}\right|}+\sum_{i=1}^{N} m_{i}^{(k)} r_{i}^{2},
$$

where we have normalized the masses and charges by that of the first $(k=1)$ species [18], and made use of the dimensionless space coordinate $r_{0}$ and energy $E_{0}$ by $r_{0}=\left(2 Q^{(1) 2} / M^{(1)} \varepsilon \omega_{0}^{2}\right)^{1 / 3}$ and $E_{0}=M^{(1)} \omega_{0}^{2} r_{0}^{2} / 2$. Without loss of generality, we have dropped the particle index $i$ on $Q_{i}^{(k)}, M_{i}^{(k)}$, $q_{i}^{(k)}$, and $m_{i}^{(k)}$, since particles of the same species are indistinguishable. For convenience, we also introduce the species mass-to-charge ratio $s^{(k)}=m^{(k)} / q^{(k)}$, such that $s^{(1)}=1$.

The model for particle trapping is applicable to a rotating system where the rotation is independent of the properties of the particles of interest. The rotation can be induced physically or as in the case of a dust-containing plasma by an external perpendicular magnetic field [23]. In the latter case, the plasma as a whole rotates at $\omega$, say the ion cyclotron frequency. The much heavier and slow-responding dust grains (of mass $m$ ) embedded in the plasma then experience a centripetal force, corresponding to the potential energy $\frac{1}{2} m \omega^{2} r^{2}$. Such a model has also been used [18] in the simulation of the self-assembly of ferromagnetic particles on a liquid-air interface $[6,7]$. There a rotating external magnetic field not only causes the fluid system to rotate but it also makes the disklike particles to spin around their own axes, resulting in a hydrodynamic Coulomb-like repulsive interaction between the particles.

We use MD simulations to follow the motion of the particles. To achieve the ground-state configuration, each run is started with random spatial and velocity distributions of the particles at a high temperature $(T=0.05 \rightarrow 1.0$, where $T$ has been normalized by $\left.E_{0}\right)$. The system is then slowly annealed until the zero temperature $\left(T=0 \pm 10^{-6}\right)$ is reached. The annealing time is $6 \times 10^{4} \omega_{0}^{-1}$, and the integration time step is $0.003 \omega_{0}^{-1}$. We also run the same systems with different random initial distributions. When possible (namely for some 2D two-species cases) our results are compared and found to agree with that obtained from Monte Carlo (MC) simulations [19]. Following Drocco et al. [17], the accuracy of the simulation was also verified by reproducing exactly the ground-state configurations of the single-species clusters $[17,22]$.

Figure 1 shows the ground-state configurations of a small two-species system with $N=19$. The subfigures, all of the same arbitrary scale, are arranged such that the columns show the effect of the relative mass-to-charge ratio $s^{(2)}$, and the rows show the effect of the relative charge $q^{(2)}$. As observed in the earlier works, Fig. 1 shows that except for the innermost ones, the particles arrange themselves into ringlike shells. For most values of $s^{(2)}$, except for $s^{(2)}=1.0$, particles of the same species are located in a common shell. Figure 1 shows that the ground-state structures depend only on $s^{(2)}$. For $s^{(2)}=0.6$, the first row in the figure shows that the particles of species 2 arrange themselves into a ringlike shell at the outer region of the trap, surrounding the particles of species 1 , and this arrangement is independent of the value of $q^{(2)}$. However, higher $q^{(2)}$ values tend to make the inner species-1 particle distribution more compact. This behavior

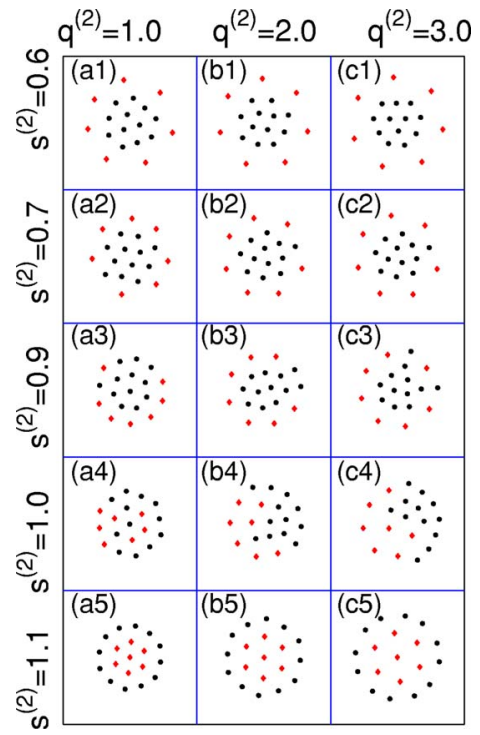

FIG. 1. (Color online) The ground-state configurations for two species of particles. The total number of particles is $N=19$. The numbers of particles of species 1 (dots) and 2 (diamonds) are $N^{(1)}=12$ and $N^{(2)}=7$, respectively.

can be expected since here the overall cluster size is almost unchanged. When the ratio $s^{(2)}$ is 0.7 , the particles of species 2 still surround that of species 1 (see the second row in Fig. 2 ), but one particle of species 1 also appears in the outer shell. When $s^{(2)}$ is increased to 0.9 , i.e., close to $s^{(1)}$, two or three particles of species 1 appear in the outer shell. That is, particles of species 1 and 2 start to compete in shell occupation. When $s^{(2)}=s^{(1)}(=1)$, the particles of the two species appear to be indistinguishable in the shell occupation, although they are of different mass and charge. When $s^{(2)}$ is further

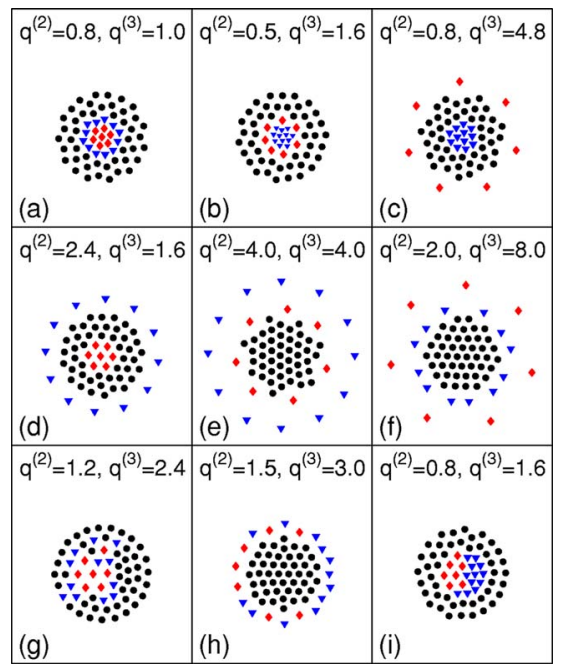

FIG. 2. (Color online) The ground-state configurations for three species of particles. The total particle number is $N=64$. The particle number of species 1 (dots) is $N^{(1)}=45$, that of species 2 (triangles) $N^{(2)}=12$, and that of species 3 (diamonds) $N^{(3)}=7$. The mass-tocharge ratios $s^{(2)}$ and $s^{(3)}$ are (a) 1.5 and 2.4, (b) 2.4 and 1.5, (c) 1.5 and 0.5 , (d) 0.5 and 1.5, (e) 0.3 and 0.6, (f) 0.6 and 0.3, (g) 1 and 1, (h) 0.8 and 0.8 , and (i) 1.5 and 1.5 , respectively. 

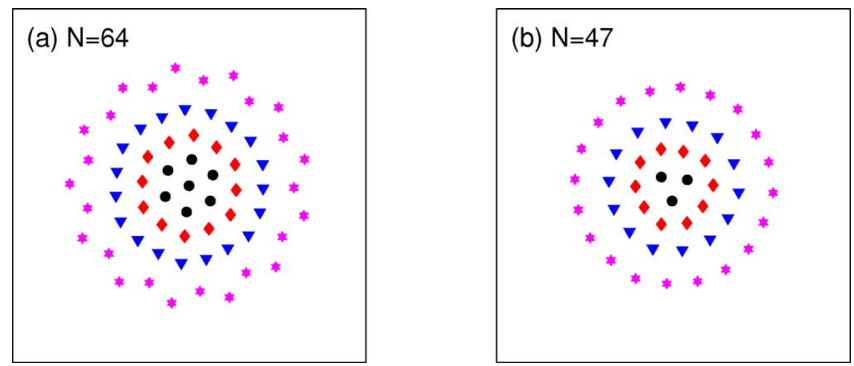

FIG. 3. (Color online) The ground-state configurations for four species of particles, for (a) $N=64, N^{(1)}=26, N^{(2)}=19, N^{(3)}=12$, and $N^{(4)}=7$; and (b) $N=47, N^{(1)}=20, N^{(2)}=14, N^{(3)}=10$, and $N^{(4)}=3$. The particles of species $1,2,3$, and 4 are denoted by asterisks, triangles, diamonds, and dots, respectively. The masses are $m^{(2)}$ $=1.2, m^{(3)}=2.4$, and $m^{(4)}=4.5$, and the charges are $q^{(2)}=0.8, q^{(3)}$ $=1.0$, and $q^{(4)}=1.5$. The corresponding mass-to-charge ratios $s^{(2)}$, $s^{(3)}$, and $s^{(4)}$ are $1.5,2.4$, and 3.0, respectively.

increased to 1.1 , i.e., larger than $s^{(1)}$, the species-2 particles are located near the center of the trap, and are surrounded by shells formed by particles of species 1 . From the above results, one can conclude that the relative locations of the particles of different species in the ground state is mainly determined by the relative mass-to-charge ratio: the larger the latter, the closer are the particles to the center of the trap [24]. Clearly, the particle packing order is determined by a delicate balance at minimum total energy of the effects of the repulsive Coulomb particle-particle interaction and the quadratic trapping potential. The latter, which is only mass dependent, tends to push the particles to the center of the trap, with the heavier ones at the bottom. On the other hand, the repulsive Coulomb interaction, which is only charge dependent, tends to prevent the particles from coming too close together. However, the actual ground-state particle ordering is determined by minimizing the total energy of the whole system.

We now verify the above conclusion for the ground-state configurations of multispecies systems. In particular, we shall consider systems with three, four, as well as five species of particles. Figure 2 shows the ground state of a system with three species. The total particle number in the system is $N=64$. For each subfigure, the mass-to-charge ratios $s^{(2)}$ and $s^{(3)}$ are different. We see again that particles of the same species tend to occupy common shells, with those of greatest mass-to-charge ratio at or near the center of the trap, and those of smallest mass-to-charge ratio at the edge of the trap. In other words, the location of the particles is still determined by the mass-to-charge ratio. However, when the ratio $s^{(2)}=s^{(3)}=s^{(1)}=1$ (i.e., when the three mass-to-charge ratios are the same), as shown in Fig. 2(g), the particles are again indistinguishable from each other and form shells in a mixed manner, despite the fact that the mass and charge of different species are different. Figure 2(h) is for $s^{(1)}>s^{(2)}=s^{(3)}=0.8$. One finds as expected that the particles of species 2 and 3 are indistinguishable from each other, and they form a ring that encloses the particles of species 1. Figure 2(i) is for $s^{(1)}<s^{(2)}=s^{(3)}=1.5$. We see that again the particles of species 2 and 3 are indistinguishable. They are at the center of the trap and surrounded

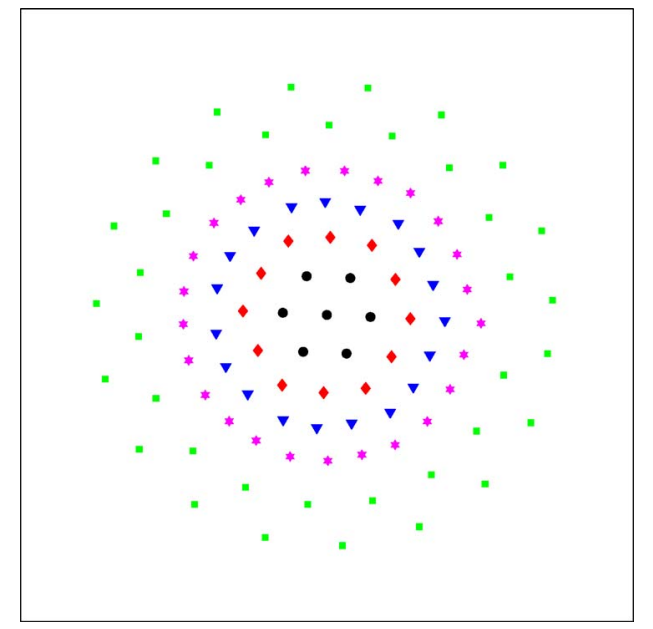

FIG. 4. (Color online) The ground-state configurations of systems with five species of particles, for $N=100, N^{(1)}=37, N^{(2)}=25$, $N^{(3)}=19, N^{(4)}=12$, and $N^{(5)}=7$. The particles from the species 1,2 , 3,4 , and 5 are denoted by squares, asterisks, triangles, diamonds, and dots, respectively. The normalized masses (charges) are $m^{(2)}=0.8\left(q^{(2)}=0.5\right), m^{(3)}=1.2\left(q^{(3)}=0.6\right), m^{(4)}=2.4\left(q^{(4)}=1.0\right)$, and $m^{(5)}=4.5\left(q^{(5)}=1.5\right)$. The corresponding mass-to-charge ratios $s^{(2)}$, $s^{(3)}, s^{(4)}$, and $s^{(5)}$ are 1.6, 2.0, 2.4, and 3.0, respectively.

by shells formed by particles of species 1 . Thus, we can see that the unique dependence of the particle distribution on the mass-to-charge ratio observed for two-species systems is valid.

Figure 3 shows the ground-state configurations of a system with four species of particles. Here we also consider the effect of the total particle number $N$. From Fig. 3(a), which is for $N=64$, one can see that the dependence of the particle distribution on the mass-to-charge ratio still applies. Figure 3 (b) is for $N=47$, comparing it with Fig. 3(a), we see that the general rule for the ground state is not affected by the total number of particles in the system. Figure 4 shows the ground state of a five-species 100-particle system. Clearly, the rule for the particle distribution again applies. We have also considered still larger and more-species systems, and found that the rule always applies. That is, the rule is indeed independent of the total particle and species numbers and is quite general. In fact, wherever possible, we have also checked for the validity of this rule in the results of existing works [25], and found that it is indeed preserved.

In conclusion, a general rule for the ground-state configuration of a multispecies system of charged particles trapped in a quadratic potential is demonstrated by means of MD simulation. It is found that the larger the mass-to-charge ratio, the closer are the particles to the center of the trap. This rule is independent of the total particle and species numbers in the system. It should be pointed out that this result is valid only for the quadratic trapping potential appearing in the potential energy term (1). From the Hamiltonian, one can see that the importance of the mass-to-charge ratio on the particle distribution is obvious for the single-component case, in fact, even during the stage of dynamical evolution. However, the importance of $s^{(k)}$ for a multispecies system is not evident. An analytical investigation, albeit rather difficult, of the 
ground-state particle distribution would thus be desirable, and the results here can be useful as a guide. Our results may also be useful for understanding many cluster and crystal models based on the quadratic trapping potential, such as in some laser-cooled ionic systems [26-28] as well as ferromagnetic particle systems [6,7].
Y.H.L. acknowledges a GOA project of the University of Antwerp for financial support and the National Science Foundation of China (Project No. 10205025) for initializing this work. Z.Y.C. would like to thank a Bilateral Project between Flanders and China for financial support.
[1] M. A. Reed and W. P. Kirk, Nanostructure Physics and Fabrication (Academic Press, Boston, 1989).

[2] P. Leiderer, W. Ebner, and V. B. Shilkin, Surf. Sci. 113, 405 (1982).

[3] Y. Kondo, J. S. Korhonen, M. Krusius, V. V. Dmitriev, E. V. Thuneberg, and G. E. Volovik, Phys. Rev. Lett. 68, 3331 (1992).

[4] R. Bubeck, C. Bechinger, S. Neser, and P. Leiderer, Phys. Rev. Lett. 82, 3364 (1999).

[5] M. Golosovsky, Y. Saado, and D. Davidov, Phys. Rev. E 65, 061405 (2002).

[6] B. A. Grzybowski, X. Jiang, H. A. Stone, and G. M. Whitesides, Phys. Rev. E 64, 011603 (2001).

[7] B. A. Grzybowski, H. A. Stone, and G. M. Whitesides, Nature (London) 405, 1033 (2000).

[8] W. T. Juan, Z. H. Huang, J. W. Hsu, Y. J. Lai, and I. Lin, Phys. Rev. E 58, R6947 (1998).

[9] Y. H. Liu, B. Liu, Y. P. Chen, S. Z. Yang, L. Wang, and X. G. Wang, Phys. Rev. E 67, 066408 (2003).

[10] B. Liu, K. Avinash, and J. Goree, Phys. Rev. E 69, 036410 (2004).

[11] Y. H. Liu, B. Liu, S. Z. Yang, and L. Wang, J. Phys. A 35, 9535 (2002).

[12] V. M. Bedanov and F. M. Peeters, Phys. Rev. B 49, 2667 (1994).

[13] V. A. Schweigert and F. M. Peeters, Phys. Rev. B 51, 7700 (1995).

[14] A. A. Koulakov and B. I. Shklovskii, Phys. Rev. B 57, 2352 (1998).

[15] I. V. Schweigert, V. A. Schweigert, and F. M. Peeters, Phys. Rev. Lett. 84, 4381 (2000).

[16] M. Block, A. Drakoudis, H. Leuthner, P. Seibert, and G. Werth, J. Phys. B 33, L375 (2000).
[17] J. A. Drocco, C. J. Olson Reichhardt, C. Reichhardt, and B. Jankó, Phys. Rev. E 68, 060401 (2003).

[18] K. Nelissen, B. Partoens, and F. M. Peeters, Phys. Rev. E 69, 046605 (2004).

[19] W. P. Ferreira, F. F. Munarin, K. Nelissen, R. N. Costa Filho, F. M. Peeters, and G. A. Farias, Phys. Rev. E 72, 021406 (2005).

[20] T. Matthey, J. P. Hansen, and M. Drewsen, Phys. Rev. Lett. 91, 165001 (2003).

[21] L. Hornekær, N. Kjærgaard, A. M. Thommesen, and M. Drewsen, Phys. Rev. Lett. 86, 1994 (2001).

[22] M. Kong, B. Partoens, and F. M. Peeters, Phys. Rev. E 65, 046602 (2002).

[23] U. Konopka, D. Samsonov, A. V. Ivlev, J. Goree, V. Steinberg, and G. E. Morfill, Phys. Rev. E 61, 1890 (2000).

[24] This result is in contrast to the expected one that larger-mass particles should be more deeply trapped since the trapping potential is only mass dependent. For example, we note that in Figs. 1(b1) and 1(c1), $m^{(2)}$ is 1.2 and 1.8 , respectively, so that here the lighter (species 1), instead of the heavier (species 2), particles are at the center of the trap. This is clearly a result of energy minimization of the system, rather than just force balance of the particles.

[25] For example, see Ref. [19]. However, the existing works considered at most two species, and also did not use the mass-tocharge ratio as a parameter. Thus the number of relevant cases that can be compared is limited.

[26] J. N. Tan, J. J. Bollinger, B. Jelenkovic, and D. J. Wineland, Phys. Rev. Lett. 75, 4198 (1995).

[27] T. B. Mitchell, J. J. Bollinger, D. H. E. Dubin, H. P. Huang, W. M. Itano, and R. H. Baughman, Science 282, 1290 (1998).

[28] T. B. Mitchell, J. J. Bollinger, W. M. Itano, and D. H. E. Dubin, Phys. Rev. Lett. 87, 183001 (2001). 